\title{
Double Copy Relation in AdS Space
}

\author{
Xinan Zhou (周稀楠) \\ Princeton Center for Theoretical Science, Princeton University, Princeton, New Jersey 08544, USA
}

(Received 27 June 2021; accepted 7 September 2021; published 27 September 2021)

\begin{abstract}
We present a double copy relation in $\mathrm{AdS}_{5}$ that relates tree-level four-point amplitudes of supergravity, super Yang-Mills, and bi-adjoint scalars.

DOI: 10.1103/PhysRevLett.127.141601
\end{abstract}

Introduction.-Scattering amplitudes in flat space exhibit surprising properties that encode deep lessons for quantum field theories and gravity. While we believe many curved-spacetime generalizations exist, explicit realizations are far from obvious to find. Recently, there has been a lot of activity trying to extend two remarkable flat-space properties, color-kinematic duality [1] and the double copy relation [2], to the simplest curved background: the anti-de Sitter (AdS) space [3-8] [9]. The flat-space relations relate gauge theory and gravity amplitudes and have numerous applications in modern amplitude research [12]. Since AdS/CFT maps AdS amplitudes to conformal field theory (CFT) correlators, generalizations to AdS are especially interesting. While color-kinematic duality has been observed for four points [5-8], AdS double copy so far has only worked for three-point functions $[3,4]$. In fact, it was not clear if the flat-space relation has to be drastically modified at higher points. In this Letter, we present an AdS generalization that realizes the double copy construction in four-point amplitudes for the first time. We relate tree-level amplitudes in $\mathrm{AdS}_{5} \times \mathrm{S}^{5}$ IIB supergravity, $\mathrm{AdS}_{5} \times \mathrm{S}^{3}$ supersymmetric Yang-Mills (SYM), and nonsupersymmetric $\mathrm{AdS}_{5} \times \mathrm{S}^{1}$ bi-adjoint scalars in a simple way that mirrors the flat-space relation. Moreover, our AdS relation works for all amplitudes in these theories, applying to massless and massive particles alike.

We will use the Mellin representation for CFT correlators $[14,15]$. AdS amplitudes become Mellin amplitudes and enjoy a simple analytic structure resembling the flat-space one. Tree-level Mellin amplitudes of AdS supergravity and super gauge theories in various spacetime dimensions were systematically studied in [7,16-23], and a Mellin colorkinematic relation similar to the flat-space one was pointed out in [7]. Unfortunately, applying the flat-space double copy prescription led to no sensible amplitudes. In this

Published by the American Physical Society under the terms of the Creative Commons Attribution 4.0 International license. Further distribution of this work must maintain attribution to the author(s) and the published article's title, journal citation, and DOI. Funded by SCOAP.
Letter, we revisit these results. We will focus on $\mathrm{AdS}_{5}$ and take advantage of supersymmetry, which allows us to reduce the Mellin amplitudes to simpler "reduced" Mellin amplitudes. We find that it is in these reduced objects that color-kinematic duality and the double copy relation are naturally realized.

Schematically, we will write the reduced amplitude of $\mathrm{AdS}_{5}$ super gluons with $\mathcal{N}=2$ superconformal symmetry as a finite sum labeled by integers $i, j$ :

$$
\widetilde{\mathcal{M}} \sim \sum_{i, j} \frac{n_{s}^{i, j} c_{s}}{s-s_{i, j}}+\frac{n_{t}^{i, j} c_{t}}{t-t_{i, j}}+\frac{n_{u}^{i, j} c_{u}}{u-u_{i, j}},
$$

where the number of terms is determined by the external masses. $c_{s, t, u}$ are standard color factors satisfying $c_{s}+c_{t}+c_{u}=0$. The kinematic factors $n_{s, t, u}^{i, j}$ turn out to obey the same relation $n_{s}^{i, j}+n_{t}^{i, j}+n_{u}^{i, j}=0$, giving rise to an AdS color-kinematic duality. Replacing $c_{s, t, u}$ with $n_{s, t, u}^{i, j}$, we recover precisely super graviton reduced amplitudes of $\mathrm{AdS}_{5} \times \mathrm{S}^{5}$ IIB supergravity $[16,17]$. On the other hand, replacing $n_{s, t, u}^{i, j}$ by $c_{s, t, u}$ leads to Mellin amplitudes of conformally coupled bi-adjoint scalars on $\mathrm{AdS}_{5} \times \mathrm{S}^{1}$, which were not studied in the literature. We will prove this fact by direct calculation. The $\mathrm{AdS}_{5}$ double copy relation presented here relates theories with varying $\mathcal{N}=0,2,4$ superconformal symmetry. However, it also implies that purely bosonic theories of Einstein gravity, Yang-Mills, and bi-adjoint scalars on $\mathrm{AdS}_{5}$ should be related by double copy, as we will briefly discuss at the end.

Four-point correlators: No supersymmetry.-Let us start with the nonsupersymmetric case. We consider the correlator of four scalar operators $\mathcal{O}_{k_{i}}$ with conformal dimensions $k_{i}$ [24]:

$$
G_{k_{1} k_{2} k_{3} k_{4}}=\left\langle\mathcal{O}_{k_{1}} \mathcal{O}_{k_{2}} \mathcal{O}_{k_{3}} \mathcal{O}_{k_{4}}\right\rangle
$$

In Mellin space, correlators are represented as [14,15]

$$
G_{k_{1} k_{2} k_{3} k_{4}}=\int_{-i \infty}^{i \infty}[d s d t] K\left(x_{i j}^{2} ; s, t, u\right) \mathcal{M}_{k_{1} k_{2} k_{3} k_{4}} \Gamma_{\left\{k_{i}\right\}}(s, t, u),
$$


where $[d s d t]=\left[d s d t /(4 \pi i)^{2}\right]$, and $K\left(x_{i j}^{2} ; s, t, u\right)$ is a factor containing all spacetime dependence:

$$
K\left(x_{i j}^{2} ; s, t, u\right)=\left(x_{12}^{2}\right)^{\frac{s-k_{1}-k_{2}}{2}}\left(x_{34}^{2}\right)^{\frac{s-k_{3}-k_{4}}{2}}\left(x_{14}^{2}\right)^{\frac{t-k_{1}-k_{4}}{2}}\left(x_{23}^{2}\right)^{\frac{t-k_{2}-k_{3}}{2}}\left(x_{13}^{2}\right)^{\frac{u-k_{1}-k_{3}}{2}}\left(x_{24}^{2}\right)^{\frac{u-k_{2}-k_{4}}{2}} .
$$

Here, $x_{i j}=x_{i}-x_{j}$, and $s, t, u$ are Mandelstam variables satisfying $s+t+u=\sum_{i=1}^{4} k_{i} \equiv \Sigma[25]$. We have also extracted a factor of Gamma functions,

$$
\Gamma_{\left\{k_{i}\right\}}(s, t, u)=\Gamma\left[\frac{k_{1}+k_{2}-s}{2}\right] \Gamma\left[\frac{k_{3}+k_{4}-s}{2}\right] \Gamma\left[\frac{k_{1}+k_{4}-t}{2}\right] \Gamma\left[\frac{k_{2}+k_{3}-t}{2}\right] \Gamma\left[\frac{k_{1}+k_{3}-u}{2}\right] \Gamma\left[\frac{k_{2}+k_{4}-u}{2}\right],
$$

that captures the contribution of double-trace operators universally present in the holographic limit [15]. All dynamic information is contained in $\mathcal{M}_{k_{1} k_{2} k_{3} k_{4}}$, known as the "Mellin amplitude." The four-point function $G_{k_{1} k_{2} k_{3} k_{4}}$ obeys Bose symmetry, which permutes operators. Bose symmetry acts on the Mellin amplitude by interchanging $k_{i}$, as well as permuting the Mandelstam variables $s, t, u$ in the same way it acts on a flat-space amplitude.

Four-point correlators: $\quad \mathcal{N}=2$ superconformal symmetry.-We now consider CFTs with $\mathcal{N}=2$ superconformal symmetry, focusing on the $\frac{1}{2}$-Bogomol'nyiPrasad-Sommerfield ( $\frac{1}{2}$-BPS) operators. These operators are of the form $\mathcal{O}_{k}^{a_{1} \ldots a_{k}}$, where $a_{i}=1,2$ are indices of the $R$-symmetry group $S U(2)_{R}$ [26]. The operator $\mathcal{O}_{k}^{a_{1} \ldots a_{k}}$ transforms in the spin $j_{R}=(k / 2)$ representation of $S U(2)_{R}$ and has conformal dimensions $k=2,3, \ldots$ To conveniently keep track of the $S U(2)_{R}$ indices, we contract them with auxiliary two-component spinors $v^{a}$ :

$$
\mathcal{O}_{k}(x, v)=\mathcal{O}_{k}^{a_{1} \ldots a_{k}} v^{b_{1}} \ldots v^{b_{k}} \epsilon_{a_{1} b_{1}} \ldots \epsilon_{a_{k} b_{k}}
$$

We then consider their four-point functions [Eq. (1)] and define the Mellin amplitude $\mathcal{M}_{k_{1} k_{2} k_{3} k_{4}}^{\mathcal{N}=2}$ via Eq. (2).

The $\mathcal{N}=2$ superconformal symmetry imposes extra constraints on the form of correlators via the superconformal Ward identities [27]. Solving them leads to

$$
G_{k_{1} k_{2} k_{3} k_{4}}^{\mathcal{N}=2}=G_{0, k_{1} k_{2} k_{3} k_{4}}^{\mathcal{N}=2}+R^{(2)} H_{k_{1} k_{2} k_{3} k_{4}}^{\mathcal{N}=2},
$$

where $G_{0, k_{1} k_{2} k_{3} k_{4}}^{\mathcal{N}=2}$ is the protected part of the correlator independent of marginal couplings. The factor $R^{(2)}$ is crossing symmetric and is fixed by superconformal symmetry to be

$$
R^{(2)}=\left(v_{1} \cdot v_{2}\right)^{2}\left(v_{3} \cdot v_{4}\right)^{2} x_{13}^{2} x_{24}^{2}(1-z \alpha)(1-\bar{z} \alpha) .
$$

Here, $v_{i} \cdot v_{j}=v_{i}^{a} v_{j}^{b} \epsilon_{a b}, \alpha=\left[\left(v_{1} \cdot v_{3}\right)\left(v_{2} \cdot v_{4}\right)\right] /\left[\left(v_{1} \cdot v_{2}\right)\left(v_{3} \cdot v_{4}\right)\right]$ is the $S U(2)_{R}$ cross ratio, and $z, \bar{z}$ are conformal cross ratios given by

$$
z \bar{z}=\frac{x_{12}^{2} x_{34}^{2}}{x_{13}^{2} x_{24}^{2}}=U, \quad(1-z)(1-\bar{z})=\frac{x_{14}^{2} x_{23}^{2}}{x_{13}^{2} x_{24}^{2}}=V .
$$

All the dynamical information is contained in the simpler "reduced" correlator $H_{k_{1} k_{2} k_{3} k_{4}}^{\mathcal{N}=2}$, which can be viewed as a correlator of operators with shifted conformal dimensions $k_{i}+1$ and shifted $S U(2)_{R}$ spins $\left(k_{i} / 2\right)-1$.

In the regime to be considered, corresponding to AdS tree level, the reduced correlator in fact captures all the information. To make it more precise, let us define a reduced Mellin amplitude via the reduced correlator

$H_{k_{1} k_{2} k_{3} k_{4}}^{\mathcal{N}=2}=\int_{-i \infty}^{i \infty}[d s d t] K\left(x_{i j}^{2} ; s, t, \tilde{u}\right) \widetilde{\mathcal{M}}_{k_{1} k_{2} k_{3} k_{4}}^{\mathcal{N}=2} \Gamma_{\left\{k_{i}\right\}}(s, t, \tilde{u})$.

Note that it is important to shift the $u$ variable to $\tilde{u}=u-2$ so that $s+t+\tilde{u}=\Sigma-2$. The shift is to compensate the nonzero weights of the factor $R^{(2)}$ under conformal transformations. As a consequence, Bose symmetry acts differently in the full and reduced Mellin amplitudes as

$$
\begin{aligned}
& \mathcal{M}_{k_{1} k_{2} k_{3} k_{4}}^{\mathcal{N}=2}: \text { permuting } s, t, u, \\
& \widetilde{\mathcal{M}}_{k_{1} k_{2} k_{3} k_{4}}^{\mathcal{N}=2}: \text { permuting } s, t, \tilde{u} .
\end{aligned}
$$

In the tree-level regime, the protected part $G_{0, k_{1} k_{2} k_{3} k_{4}}^{\mathcal{N}=2}$ does not contribute to the Mellin amplitude [7]. Rather it is generated by a contour pinching mechanism described in [17]. Therefore, full amplitudes are completely determined by reduced amplitudes, with the precise relation given by translating both sides of Eq. (5) into Mellin space

$$
\mathcal{M}_{k_{1} k_{2} k_{3} k_{4}}^{\mathcal{N}=2}=\mathbb{R}^{(2)} \circ \widetilde{\mathcal{M}}_{k_{1} k_{2} k_{3} k_{4}}^{\mathcal{N}=2} .
$$

The factor $R^{(2)}$ now becomes a difference operator $\mathbb{R}^{(2)}$ [7]. To obtain it, we interpret each monomial $U^{m} V^{n}$ in

$$
\frac{R^{(2)}}{\left(v_{1} \cdot v_{2}\right)^{2}\left(v_{3} \cdot v_{4}\right)^{2} x_{13}^{2} x_{24}^{2}}
$$

as a difference operator $U^{m} V^{n} \rightarrow \mathbb{O}_{m, n}^{(2)}$ that acts on functions $f(s, t)$ according to 


$$
\mathbb{O}_{m, n}^{(\mathcal{N})} \circ f(s, t)=\frac{\Gamma_{\left\{k_{i}\right\}}(s-2 m, t-2 n, \Sigma-\mathcal{N}-s-t+2 m+2 n)}{\Gamma_{\left\{k_{i}\right\}}(s, t, \Sigma-s-t)} \times f(s-2 m, t-2 n) .
$$

Four-point correlators: $\mathcal{N}=4$ superconformal symmetry.-The kinematics of $\mathcal{N}=4$ is similar. The $\frac{1}{2}$ BPS operator, labeled by an integer $k=2,3, \ldots$, transforms in the rank- $k$ symmetric traceless representation of the $S O(6)_{R} R$-symmetry group and has dimension $k$. We keep track of the $R$-symmetry indices by using null $S O(6)$ vectors $t^{r}$ [28]:

$$
O_{k}(x, t)=O^{r_{1} \ldots r_{k}}(x) t^{r_{1}} \ldots t^{r_{k}}, \quad r_{i}=1, \ldots, 6,
$$

where $t \cdot t=0$. The $\mathcal{N}=4$ superconformal symmetry dictates that the four-point function is of the form [27,29]

$$
G_{k_{1} k_{2} k_{3} k_{4}}^{\mathcal{N}=4}=G_{0, k_{1} k_{2} k_{3} k_{4}}^{\mathcal{N}=4}+R^{(4)} H_{k_{1} k_{2} k_{3} k_{4}}^{\mathcal{N}=4},
$$

where $G_{0, k_{1} k_{2} k_{3} k_{4}}^{\mathcal{N}=4}$ is the protected part, and $H_{k_{1} k_{2} k_{3} k_{4}}^{\mathcal{N}=4}$ is the reduced correlator. Note that the reduced correlator also has shifted quantum numbers, with dimensions $k_{i}+2$ and $S O(6)$ spin $k_{i}-2$ for each operator. The factor $R^{(4)}$ is determined by supersymmetry

$R^{(4)}=t_{12}^{2} t_{34}^{2} x_{13}^{4} x_{24}^{4}(1-z \alpha)(1-\bar{z} \alpha)(1-z \bar{\alpha})(1-\bar{z} \bar{\alpha})$

and doubles the $\mathcal{N}=2$ factor [Eq. (6)]. Here $t_{i j}=t_{i} \cdot t_{j}$, and

$\alpha \bar{\alpha}=\frac{t_{13} t_{24}}{t_{12} t_{34}}=\sigma, \quad(1-\alpha)(1-\bar{\alpha})=\frac{t_{14} t_{23}}{t_{12} t_{34}}=\tau$.

The full correlator $G_{k_{1} k_{2} k_{3} k_{4}}^{\mathcal{N}=4}$ gives rise to the full amplitude $\mathcal{M}_{k_{1} k_{2} k_{3} k_{4}}^{\mathcal{N}=4}$ via Eq. (2). The $\mathcal{N}=4$ reduced amplitude is similarly given by

$H_{k_{1} k_{2} k_{3} k_{4}}^{\mathcal{N}=4}=\int_{-i \infty}^{i \infty}[d s d t] K\left(x_{i j}^{2} ; s, t, \tilde{u}\right) \widetilde{\mathcal{M}}_{k_{1} k_{2} k_{3} k_{4}}^{\mathcal{N}=4} \Gamma_{\left\{k_{i}\right\}}(s, t, \tilde{u})$.

But note here that the shift in $\tilde{u}$ is by 4 , i.e., $\tilde{u}=u-4$. The greater shift is due to the higher conformal weights of $R^{(4)}$. Bose symmetry again permutes $s, t, u$ in $\mathcal{M}_{k_{1} k_{2} k_{3} k_{4}}^{\mathcal{N}=4}$ and $s, t$, $\tilde{u}$ in $\widetilde{\mathcal{M}}_{k_{1} k_{2} k_{3} k_{4}}^{\mathcal{N}=4}$. At AdS tree level, the protected part again does not contribute to the Mellin amplitude [16,17]. Therefore, the full amplitudes are determined by the reduced amplitudes via

$$
\mathcal{M}_{k_{1} k_{2} k_{3} k_{4}}^{\mathcal{N}=4}=\mathbb{R}^{(4)} \circ \widetilde{\mathcal{M}}_{k_{1} k_{2} k_{3} k_{4}}^{\mathcal{N}=4}
$$

where we have promoted $R^{(4)}$ into a difference operator $\mathbb{R}^{(4)}[16,17]$. The action of each monomial $U^{m} V^{n}$ in $R^{(4)} /\left[\left(t_{12}\right)^{2}\left(t_{34}\right)^{2} x_{13}^{4} x_{24}^{4}\right]$ is given by Eq. (11) with $\mathcal{N}=4$.
Super gluon amplitudes.-We are now ready to discuss holographic correlators in specific theories. We start with super gluons in $\mathrm{AdS}_{5}$ preserving $\mathcal{N}=2$ superconformal symmetry, which can be realized as D3 branes probing F theory singularities [30,31], or as $\mathcal{N}=4$ SYM with probe flavor D7 branes [32]. In both case, there is an $\mathrm{AdS}_{5} \times \mathrm{S}^{3}$ subspace in the holographic description, on which live localized degrees of freedom transforming in the adjoint representation of a color group $G_{F}$. These degrees of freedom form a vector multiplet, and its Kaluza-Klein reduction gives infinite towers of $\frac{1}{2}$-BPS superconformal multiplets. We refer to the $\frac{1}{2}$-BPS superprimaries as super gluons. At large central charge, gravity decouples and one has only a spin-1 gauge theory. Note $S^{3}$ has isometry $S O(4)=S U(2)_{R} \times S U(2)_{L}$. The first factor is identified with the $\mathcal{N}=2 R$-symmetry group, while the second $S U(2)_{L}$ is a global symmetry suppressed in the above discussion. The operator $\mathcal{O}_{k}$ has spin $[(k-2) / 2]$ under $S U(2)_{L}$ [31]. We can similarly contract the indices with $k-2 S U(2)_{L}$ spinors $\bar{v}^{\bar{a}}, \bar{a}=1,2$. In reduced correlators, $v$ and $\bar{v}$ further recombine into null vectors of $S O(4)$ via Pauli matrices and appear only as polynomials of $t_{i j}$ [7]:

$$
t^{r^{\prime}}=\sigma_{a \bar{a}}^{r^{\prime}} v^{a} \bar{v}^{\bar{a}}, \quad r^{\prime}=1, \ldots, 4, \quad t \cdot t=0 .
$$

To write down the super gluon amplitudes, let us choose, without loss of generality, the ordering $k_{1} \leq k_{2} \leq k_{3} \leq k_{4}$, and distinguish two cases:

$$
k_{1}+k_{4} \geq k_{2}+k_{3}(\text { case I }), \quad k_{1}+k_{4}<k_{2}+k_{3}(\text { case II }) .
$$

To measure the deviation from the equal weight case $k_{i}=(\Sigma / 4)$, it is useful to introduce the following parameters:

$\kappa_{s}=\left|k_{3}+k_{4}-k_{1}-k_{2}\right|, \quad \kappa_{t}=\left|k_{1}+k_{4}-k_{2}-k_{3}\right|$,

$\kappa_{u}=\left|k_{1}+k_{3}-k_{2}-k_{4}\right|$.

The reduced Mellin amplitudes are given by $[7,33]$

$$
\begin{aligned}
\widetilde{\mathcal{M}}_{k_{1} k_{2} k_{3} k_{4}}^{\mathcal{N}=2}= & \sum_{\substack{i+j+k=\mathcal{E}-2 \\
0 \leq i, j, k \leq \mathcal{E}-2}} \frac{\sigma^{i} \tau^{j}}{i ! j ! k !\left(\frac{2 i+\kappa_{u}}{2}\right) !\left(\frac{2 j+\kappa_{t}}{2}\right) !\left(\frac{2 k+\kappa_{s}}{2}\right) !} \\
& \times\left[\frac{n_{s}^{i, j} c_{s}}{s-s_{M}+2 k}+\frac{n_{t}^{i, j} c_{t}}{t-t_{M}+2 j}+\frac{n_{u}^{i, j} c_{u}}{\tilde{u}-u_{M}+2 i}\right] \\
& \times I\left(t_{a b}\right),
\end{aligned}
$$

which has been rewritten to manifest Bose symmetry. Let us unpack this expression a bit. Here, 


$$
\mathcal{E}=\frac{k_{1}+k_{2}+k_{3}-k_{4}}{2}(\text { case I }), \quad \mathcal{E}=k_{1}(\text { case II })
$$

is the "extremality," which determines the complexity of the amplitude. After extracting a factor in $t_{a b}$,

$$
I\left(t_{a b}\right)=t_{34}^{\frac{\kappa_{s}}{2}} t_{24}^{\frac{\kappa_{u}}{2}}\left(t_{12} t_{34}\right)^{-\mathcal{E}+2} \times \begin{cases}\frac{\kappa_{t}}{t_{14}^{2}} & (\text { case I }) \\ t_{23}^{\frac{\kappa_{t}}{2}} & (\text { case II) }\end{cases}
$$

the reduced Mellin amplitudes are degree- $(\mathcal{E}-2)$ polynomials in $\sigma$ and $\tau$ defined in Eq. (15). The color dependence is captured by the color factors

$$
c_{s}=f^{I_{1} I_{2} J} f^{J I_{3} I_{4}}, \quad c_{t}=f^{I_{1} I_{4} J} f^{J I_{2} I_{3}}, \quad c_{u}=f^{I_{1} I_{3} J} f^{J I_{2} I_{4}},
$$

where $f^{I J K}$ are the structure constants of the color group $G_{F}$. Thanks to the Jacobi identity, they satisfy $c_{s}+c_{t}+c_{u}=0$. The kinematic factors $n_{s, t, u}^{i, j}$ are given by

$$
\begin{aligned}
& n_{s}^{i, j}=\frac{1}{t-t_{M}+2 j}-\frac{1}{\tilde{u}-u_{M}+2 i}, \\
& n_{t}^{i, j}=\frac{1}{\tilde{u}-u_{M}+2 i}-\frac{1}{s-s_{M}+2 k}, \\
& n_{u}^{i, j}=\frac{1}{s-s_{M}+2 k}-\frac{1}{t-t_{M}+2 j} .
\end{aligned}
$$

The nonlocality of these expressions is only superficial and should not raise any alarm. In fact, a similar phenomenon occurs in flat space [34]. Evidently, $n_{s, t, u}^{i, j}$ obey

$$
n_{s}^{i, j}+n_{t}^{i, j}+n_{u}^{i, j}=0
$$

which gives rise to a realization of the "color-kinematic duality" [1] in AdS. In contrast to the duality pointed out in [7], this new realization has the same form for both massless $\left(k_{i}=2\right)$ and massive $\left(k_{i}>2\right)$ super gluons. Finally, the remaining parameters are given by

$$
\begin{aligned}
s_{M} & =\min \left\{k_{1}+k_{2}, k_{3}+k_{4}\right\}-2, \\
t_{M} & =\min \left\{k_{1}+k_{4}, k_{2}+k_{3}\right\}-2, \\
u_{M} & =\min \left\{k_{1}+k_{3}, k_{2}+k_{4}\right\}-2 .
\end{aligned}
$$

Super graviton amplitudes.-Let us now take a further step with the color-kinematic duality [Eq. (21)] and replace color factors $c_{s, t, u}$ in each monomial $\sigma^{i} \tau^{j}$ by kinematic factors $n_{s, t, u}^{i, j}$. The result is

$$
\begin{aligned}
\widetilde{\mathcal{M}}_{k_{1} k_{2} k_{3} k_{4}}^{\mathcal{N}=2 \otimes \mathcal{N}=2}= & \sum_{\substack{i+j+k=\mathcal{E}-2 \\
0 \leq i, j, k \leq \mathcal{E}-2}} \frac{\sigma^{i} \tau^{j}}{i ! j ! k !\left(\frac{2 i+\kappa_{u}}{2}\right) !\left(\frac{2 j+\kappa_{t}}{2}\right) !\left(\frac{2 k+\kappa_{s}}{2}\right) !} \\
& \times \frac{-9 I\left(t_{a b}\right)}{\left(s-s_{M}+2 k\right)\left(t-t_{M}+2 j\right)\left(\tilde{u}-u_{M}+2 i\right)} .
\end{aligned}
$$

To interpret it as $\mathcal{N}=4$ reduced amplitudes, we need to replace the $\tilde{u}$ variable with the $\mathcal{N}=4$ one, as required by Bose symmetry of $\widetilde{\mathcal{M}}_{k_{1} k_{2} k_{3} k_{4}}^{\mathcal{N}=4}$. Furthermore, we replace the $S O(4)$ vectors $t^{r^{\prime}}$ by $S O(6)$ null vectors [35]. Remarkably, it gives all the super graviton reduced Mellin amplitudes of IIB supergravity on $\mathrm{AdS}_{5} \times \mathrm{S}^{5}[16,17]$

$$
\widetilde{\mathcal{M}}_{k_{1} k_{2} k_{3} k_{4}}^{\mathcal{N}=4}=\sqrt{k_{1} k_{2} k_{3} k_{4}} \times \widetilde{\mathcal{M}}_{k_{1} k_{2} k_{3} k_{4}}^{\mathcal{N}=2 \otimes \mathcal{N}=2}
$$

up to an overall factor [36]. This generalizes the "double copy relation" [2] into AdS space for four-point functions [38]. In fact, redefining the super gravitons by $\mathcal{O}_{k} \rightarrow$ $\mathcal{O}_{k} / \sqrt{k}$ gets rid of the normalization factor and gives the super graviton three-point functions also as the square of the super gluon ones [39].

Bi-adjoint scalar amplitudes. - In flat space, one can also replace kinematic factors by color factors and obtain amplitudes of bi-adjoint scalars. We show that the same happens in AdS, and it serves as a nontrivial check. Note that in the above example the superconformal factor $R^{(2)}$ was doubled to $R^{(4)}$ [c.f. Eqs. (6) and (14)]. Going in the opposite direction, we expect $R^{(0)}=1$, i.e., the resulting theory has no supersymmetry. Moreover, since the internal spaces changed from $\mathrm{S}^{3}$ to $\mathrm{S}^{5}$, a reasonable guess is that this sequence starts with $S^{1}$, which will soon be confirmed. The symmetry groups are therefore $S O(\mathcal{N}+2)$, and we recall that operators in the reduced amplitudes transform in the rank- $\left(k_{i}-2\right)$ symmetric traceless representation.

Note that for $\mathcal{N}=0$, the null polarization vectors are two-component. Since we can rescale the null vectors, we are left with two inequivalent choices:

$$
t_{ \pm}=\frac{1}{\sqrt{2}}(1, \pm i)
$$

The dimension $k$ operator $\mathcal{O}_{k}^{ \pm} \equiv \mathcal{O}_{k}\left(x, t_{ \pm}\right)$has $\pm(k-2)$ charges under $U(1)=S O(2)$, depending on the polarization chosen. Moreover, we assume the scalar interactions are only cubic. Then, $U(1)$ charge conservation dictates that at least one of the $\kappa_{s}, \kappa_{t}, \kappa_{u}$ parameters in Eq. (18) is zero. For the chosen ordering $k_{1} \leq k_{2} \leq k_{3} \leq k_{4}$, we must impose the condition $\kappa_{t}=0$. This leaves

$$
\left\langle\mathcal{O}_{k_{1}}^{+} \mathcal{O}_{k_{2}}^{-} \mathcal{O}_{k_{3}}^{-} \mathcal{O}_{k_{4}}^{+}\right\rangle, \quad \text { or } \quad\left\langle\mathcal{O}_{k_{1}}^{-} \mathcal{O}_{k_{2}}^{+} \mathcal{O}_{k_{3}}^{+} \mathcal{O}_{k_{4}}^{-}\right\rangle,
$$

which have identical amplitudes [41]. Noting 


$$
\sigma=1, \quad \tau=0, \quad \mathrm{I}\left(t_{a b}\right)=1
$$

and replacing $n_{s, t, u}^{i j}$ with the color factors $c_{s, t, u}^{\prime}$ for another color group $G_{F}^{\prime}$, we find

$$
\begin{aligned}
\mathcal{M}_{k_{1} k_{2} k_{3} k_{4}}^{\mathcal{N}=0}= & \sum_{\substack{i+k=\mathcal{E}-2 \\
0 \leq i, k \leq \mathcal{E}-2}} \frac{-2 \mathcal{N}_{k_{1} k_{2} k_{3} k_{4}}}{i ! k !\left(\frac{2 i+\kappa_{u}}{2}\right) !\left(\frac{2 k+\kappa_{s}}{2}\right) !} \\
& \times\left[\frac{c_{s} c_{s}^{\prime}}{s-s_{M}+2 k}+\frac{c_{t} c_{t}^{\prime}}{t-t_{M}}+\frac{c_{u} c_{u}^{\prime}}{u-u_{M}+2 i}\right] .
\end{aligned}
$$

We dropped the tildes because nonsupersymmetric theories have only full amplitudes [Eq. (2)], and there is no shift in the $u$ variable. We also included a to-be-determined $k_{i^{-}}$ dependent normalization factor $-2 \mathcal{N}_{k_{1} k_{2} k_{3} k_{4}}$ as in the supergravity case. Remarkably, Eq. (27) can be rewritten as the sum of three $\mathrm{AdS}_{5}$ scalar exchange diagrams:

$\mathcal{N}_{k_{1} k_{2} k_{3} k_{4}}\left[\frac{c_{s} c_{s}^{\prime}}{p_{s}-1} \mathcal{S}_{p_{s}}^{(s)}+\frac{c_{t} c_{t}^{\prime}}{p_{t}-1} \mathcal{S}_{p_{t}}^{(t)}+\frac{c_{u} c_{u}^{\prime}}{p_{u}-1} \mathcal{S}_{p_{u}}^{(u)}\right]$

where $\mathcal{S}_{p}^{(s)}$ is the amplitude of exchanging a dimension- $p$ scalar in the $s$-channel (and similarly for the other two channels) [42]:

$$
\begin{aligned}
\mathcal{S}_{p}^{(s)}= & \sum_{m=0}^{\infty} \frac{-2\left(\frac{2+p-k_{1}-k_{2}}{2}\right)_{m}\left(\frac{2+p-k_{3}-k_{4}}{2}\right)_{m}}{(s-p-2 m) m !(p-1)_{m} \Gamma\left[\frac{k_{1}+k_{2}-p}{2}\right] \Gamma\left[\frac{k_{3}+k_{4}-p}{2}\right]} \\
& \times \frac{\Gamma[p]}{\Gamma\left[\frac{k_{1}-k_{2}+p}{2}\right] \Gamma\left[\frac{k_{2}-k_{1}+p}{2}\right] \Gamma\left[\frac{k_{3}-k_{4}+p}{2}\right] \Gamma\left[\frac{k_{4}-k_{3}+p}{2}\right]} .
\end{aligned}
$$

Moreover, the weights $p_{s, t, u}$ are precisely those selected by the $U(1)$ charge conservation

$$
p_{s}=k_{2}-k_{1}+2, \quad p_{t}=k_{1}+k_{4}-2, \quad p_{u}=k_{3}-k_{1}+2 .
$$

Note that Eq. (27) is equivalent to Eq. (28), is highly nontrivial, and a priori does not need to happen. We can further fix the normalization $\mathcal{N}_{k_{1} k_{2} k_{3} k_{4}}$ by noting $\mathcal{N}_{k_{1} k_{2} k_{3} k_{4}} /\left(p_{s}-1\right)$, etc., have the interpretation of products of three-point function coefficients $C_{k_{1} k_{2} p_{s}} C_{k_{3} k_{4} p_{s}}$. The solution, up to a $k_{i}$-independent overall factor, is

$$
C_{k_{1} k_{2} k_{3}}=\frac{1}{\sqrt{\left(k_{1}-1\right)\left(k_{2}-1\right)\left(k_{3}-1\right)}} .
$$

Finally, we confirm by direct calculation that the theory is conformally coupled scalars on $\operatorname{AdS}_{5} \times \mathrm{S}^{1}$. The conformal mass on this manifold is $M_{\text {conf }}^{2}=-4$ [43]. Decomposing the scalar field $\phi$ into $S^{1}$ modes $\phi(z, \tau)=\sum_{n=-\infty}^{\infty} \varphi_{n}(z) e^{i n \tau}$, we find each mode has mass $M_{n}^{2}=n^{2}-4$. This translates into a conformal dimension $|n|+2$, agreeing with our charge-dimension relation $n= \pm(k-2)$. We can further check three-point functions. A cubic vertex $\phi^{3}$ in $\mathrm{AdS}_{5} \times \mathrm{S}^{1}$ gives rise to infinitely many $\mathrm{AdS}_{5}$ cubic vertices $\sum \varphi_{n_{1}} \varphi_{n_{2}} \varphi_{n_{3}}$, where $\left\{n_{i}\right\}$ conserve the $U(1)$ charge. Using the result of [44], it is straightforward to show that three-point functions are precisely as in Eq. (29). Note that both $C_{k_{1} k_{2} k_{3}}$ and $\mathcal{N}_{k_{1} k_{2} k_{3} k_{4}}$ can be set to one by redefining $\mathcal{O}_{k} \rightarrow \sqrt{k-1} \mathcal{O}_{k}$. Then, the double copy relation also holds for three-point functions.

Discussions.-In this Letter, we found an extension of the double copy relation in curved spacetimes that relates all tree-level four-point functions of $\mathrm{AdS}_{5} \times \mathrm{S}^{5}$ IIB supergravity, $\mathrm{AdS}_{5} \times \mathrm{S}^{3} \mathrm{SYM}$, and $\mathrm{AdS}_{5} \times \mathrm{S}^{1}$ bi-adjoint scalars. Although our result is supersymmetric, it has immediate implications on bosonic Einstein gravity and Yang-Mills theory in $\mathrm{AdS}_{5}$ with no internal factor. Thanks to supersymmetry, four-graviton and four-gluon amplitudes can be obtained from the reduced correlators of $k_{i}=2$ super gravitons and super gluons by action of differential operators [45]. At tree level, these spinning correlators are identical to the ones in bosonic theories because the exchanged fields are the same [46]. Our result, then, indicates that the bosonic amplitudes should also be related by a double copy construction [47], the details of which we will leave for a future work. Another interesting direction is to extend our results to higher points, although more data on holographic correlators is needed [48]. While the focus here is $\mathrm{AdS}_{5}$ amplitudes, double copy relations for other backgrounds are also worth exploring. In particular, the $\mathrm{AdS}_{7}$ case [21] admits similar definitions of reduced amplitudes [50,51]. Finally, it would be interesting to explore extensions at higher genus, where the relevant CFT techniques were developed in [52].

I thank Fernando Alday, Henrik Johansson, and Lionel Mason for very helpful comments on the manuscript. This work is supported in part by the Simons Foundation Grant No. 488653.

[1] Z. Bern, J. J. M. Carrasco, and H. Johansson, New relations for gauge-theory amplitudes, Phys. Rev. D 78, 085011 (2008).

[2] Z. Bern, John Joseph M. Carrasco, and H. Johansson, Perturbative Quantum Gravity as a Double Copy of Gauge Theory, Phys. Rev. Lett. 105, 061602 (2010).

[3] J. A. Farrow, A. E. Lipstein, and P. McFadden, Double copy structure of CFT correlators, J. High Energy Phys. 02 (2019) 130.

[4] A. E. Lipstein and P. McFadden, Double copy structure and the flat space limit of conformal correlators in even dimensions, Phys. Rev. D 101, 125006 (2020).

[5] C. Armstrong, A. E. Lipstein, and J. Mei, Color/kinematics duality in $\mathrm{AdS}_{4}$, J. High Energy Phys. 02 (2021) 194. 
[6] S. Albayrak, S. Kharel, and D. Meltzer, On duality of color and kinematics in (A)dS momentum space, J. High Energy Phys. 03 (2021) 249.

[7] L. F. Alday, C. Behan, P. Ferrero, and X. Zhou, Gluon scattering in AdS from CFT, J. High Energy Phys. 06 (2021) 020.

[8] P. Diwakar, A. Herderschee, R. Roiban, and F. Teng, BCJ amplitude relations for anti-de sitter boundary correlators in embedding space, arXiv:2106.10822.

[9] See, e.g., Refs. [10,11] for progress in other curved backgrounds.

[10] T. Adamo, E. Casali, L. Mason, and S. Nekovar, Scattering on plane waves and the double copy, Classical Quantum Gravity 35, 015004 (2018).

[11] T. Adamo, E. Casali, L. Mason, and S. Nekovar, Plane wave backgrounds and colour-kinematics duality, J. High Energy Phys. 02 (2019) 198.

[12] See Ref. [13] for a recent comprehensive review.

[13] Z. Bern, J. J. Carrasco, M. Chiodaroli, H. Johansson, and R. Roiban, The duality between color and kinematics and its applications, arXiv:1909.01358.

[14] G. Mack, D-independent representation of conformal field theories in D dimensions via transformation to auxiliary dual resonance models. Scalar amplitudes, arXiv: 0907.2407.

[15] J. Penedones, Writing CFT correlation functions as AdS scattering amplitudes, J. High Energy Phys. 03 (2011) 025.

[16] L. Rastelli and X. Zhou, Mellin Amplitudes for $\mathrm{AdS}_{5} \times \mathrm{S}^{5}$, Phys. Rev. Lett. 118, 091602 (2017).

[17] L. Rastelli and X. Zhou, How to succeed at holographic correlators without really trying, J. High Energy Phys. 04 (2018) 014.

[18] L. Rastelli, K. Roumpedakis, and X. Zhou, $\mathbf{A d S}_{3} \times \mathbf{S}^{3}$ treelevel correlators: Hidden six-dimensional conformal symmetry, J. High Energy Phys. 10 (2019) 140.

[19] S. Giusto, R. Russo, A. Tyukov, and C. Wen, Holographic correlators in $\mathrm{AdS}_{3}$ without Witten diagrams, J. High Energy Phys. 09 (2019) 030.

[20] S. Giusto, R. Russo, A. Tyukov, and C. Wen, The CFT 6 origin of all tree-level 4-point correlators in $\mathrm{AdS}_{3} \times \mathrm{S}^{3}$, Eur. Phys. J. C 80, 736 (2020).

[21] L. F. Alday and X. Zhou, All Tree-Level Correlators for MTheory on $\mathrm{AdS}_{7} \times \mathrm{S}^{4}$, Phys. Rev. Lett. 125, 131604 (2020).

[22] L. F. Alday and X. Zhou, All Holographic Four-Point Functions in All Maximally Supersymmetric CFTs, Phys. Rev. X 11, 011056 (2021).

[23] C. Wen and S.-Q. Zhang, Notes on gravity multiplet correlators in $\mathrm{AdS}_{3} \times \mathrm{S}^{3}$, J. High Energy Phys. 07 (2021) 125.

[24] In this paper, the two-point functions of all $\mathcal{O}_{k}$ are unit normalized.

[25] Note that only two of the three Mandelstam variables are independent. While rewriting a function of $s, t$ in terms of $s$, $t, u$ is not unique, there is no ambiguity in defining expressions using all three variables.

[26] There is also a $U(1)_{r} R$-symmetry in the $\mathcal{N}=2$ superconformal algebra but the $\frac{1}{2}$-BPS operators are not charged under $U(1)_{r}$.

[27] M. Nirschl and H. Osborn, Superconformal Ward identities and their solution, Nucl. Phys. B711, 409 (2005).
[28] These null vectors automatically project the indices to the symmetric traceless representation.

[29] B. Eden, A. C. Petkou, C. Schubert, and E. Sokatchev, Partial nonrenormalization of the stress tensor four point function in $N=4 \mathrm{SYM}$ and AdS/CFT, Nucl. Phys. B607, 191 (2001).

[30] A. Fayyazuddin and M. Spalinski, Large N superconformal gauge theories and supergravity orientifolds, Nucl. Phys. B535, 219 (1998).

[31] O. Aharony, A. Fayyazuddin, and J. M. Maldacena, The Large $\mathrm{N}$ limit of $N=2, N=1$ field theories from three-branes in F theory, J. High Energy Phys. 07 (1998) 013.

[32] A. Karch and E. Katz, Adding flavor to AdS/CFT, J. High Energy Phys. 06 (2002) 043.

[33] Here, we have set the gauge coupling to a convenient value that does not affect the physics.

[34] In flat-space $\mathcal{N}=4 \mathrm{SYM}$, the four-point superamplitude at tree level can be similarly written as $\delta^{4}(Q) \delta^{4}(\tilde{Q})\left[\left(c_{s} n_{s} / 2\right)+\left(c_{t} n_{t} / t\right)+\left(c_{u} n_{u} / u\right)\right] . \delta^{4}(Q) \delta^{4}(\tilde{Q})$ is roughly identified with $R^{(2)}$, and the numerators $n_{s}=\frac{1}{3}[(1 / t)-(1 / u)], \quad n_{t}=\frac{1}{3}[(1 / u)-(1 / s)], \quad n_{u}=$ $\frac{1}{3}[(1 / s)-(1 / t)]$ are similarly nonlocal. However, this nonlocality is merely an artifact of manifesting all the supersymmetry. We thank H. Johansson for comments on this.

[35] Note that the dimensionality of the $S O$ group is invisible in scalar products $t_{a b}$.

[36] The $k_{i}$-dependent normalization factor was fixed in Ref. [37]. We have also set the Newton constant to a convenient value.

[37] F. Aprile, J. Drummond, P. Heslop, and H. Paul, The double-trace spectrum of $N=4$ SYM at strong coupling, Phys. Rev. D 98, 126008 (2018).

[38] In flat space, the four-point superamplitude of $\mathcal{N}=8$ supergravity has the form $\delta^{8}(Q) \delta^{8}(\tilde{Q})[(-1) / s t u]$, where the doubled supercharge delta functions correspond to $R^{(4)}$. Note the supergravity amplitude is also related to the flatspace SYM amplitude in Ref. [34] by the double copy relation $c_{s, t, u} \rightarrow n_{s, t, u}$.

[39] The new normalization makes the three-point function coefficients of super gravitons [40] independent of $k_{i}$, i.e., $C_{k_{1} k_{2} k_{3}} \sim 1$. The super gluons have exactly the same three-point functions [7].

[40] S. Lee, S. Minwalla, M. Rangamani, and N. Seiberg, Three point functions of chiral operators in $\mathrm{D}=4, \mathrm{~N}=4 \mathrm{SYM}$ at large N, Adv. Theor. Math. Phys. 2, 697 (1998).

[41] They are the charge conjugation of each other.

[42] Here, the normalization is such that the scalar operator $p$ has a unit operator product expansion coefficient.

[43] We have set $R_{\text {AdS }}=R_{\mathrm{S}}=1$.

[44] D. Z. Freedman, S. D. Mathur, A. Matusis, and L. Rastelli, Correlation functions in the $\mathrm{CFT}(\mathrm{d}) / \mathrm{AdS}(\mathrm{d}+1)$ correspondence, Nucl. Phys. B546, 96 (1999).

[45] G. P. Korchemsky and E. Sokatchev, Four-point correlation function of stress-energy tensors in $\mathcal{N}=4$ superconformal theories, J. High Energy Phys. 12 (2015) 133.

[46] This follows directly from the symmetry selection rules.

[47] The kinematic numerators in this case will likely admit a local form, but they will be gauge-dependent. 
[48] The five-point function of $\mathrm{AdS}_{5}$ massless super gravitons has been computed in Ref. [49].

[49] V. Gonçalves, R. Pereira, and X. Zhou, 20' five-point function from $\mathrm{AdS}_{5} \times \mathrm{S}^{5}$ supergravity, J. High Energy Phys. 10 (2019) 247.

[50] L. Rastelli and X. Zhou, Holographic four-point functions in the $(2,0)$ theory, J. High Energy Phys. 06 (2018) 087.
[51] X. Zhou, On superconformal four-point Mellin amplitudes in dimension $d>2$, J. High Energy Phys. 08 (2018) 187.

[52] O. Aharony, L. F. Alday, A. Bissi, and E. Perlmutter, Loops in AdS from conformal field theory, J. High Energy Phys. 07 (2017) 036. 